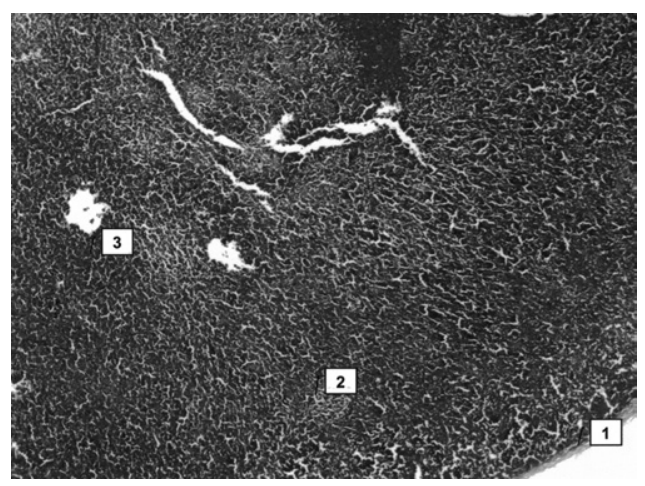

Figure 2. Histologic study of the resected specimen. 1, Capsule; 2, white pulp; 3, red pulp.

taly, most splenic nodules are mistaken for pleural parenchymal neoplastic lesions. Video-assisted thoracic surgery or thoracotomy is usually required to set the diagnosis.
The computed tomographic scan shows nonspecific round or ovoid masses with well-defined borders. Their location is variable. A splenule enhances homogeneously on contrast-enhanced images to a similar density as that of the spleen. Magnetic resonance, along with the administration of super-paramagnetic iron oxide, may be helpful and results in loss of signal intensity in all pulse sequences, similar to that seen within normal spleen. A radiologic diagnosis can be confirmed by either a technetium sulfur colloid, iodine-labeled, or technetium heat-damaged erythrocyte study, which all result in increased uptake of the radioactive isotope in the ectopic splenic tissue.

\section{References}

1. Balacumaraswami L, Yeatman M, Ghosh AK, Collins C, ForresterWood CP. Accessory spleniculi in the right hemithorax. Ann Thorac Surg. 2002;74:2172-41.

2. Madjar S, Weissberg D. Thoracic splenosis. Thorax. 1994;49: 1020-2.

3. Lee HJ, Kim YT, Kang CH, Kim JH. An accessory spleen misrecognized as an intrathoracic mass. Eur J Cardiothorac Surg. 2005;28:640.

\title{
Interventional closure of postpneumonectomy bronchial pleural fistula with a self-expandable double umbrella-shaped occluder knitted with nitinol shape memory alloy
}

Jianhua Zhang, MD, PhD, ${ }^{a}$ Shengshou $\mathrm{Hu}, \mathrm{MD}, \mathrm{PhD},{ }^{\mathrm{b}}$ Bingren Gao, MD, PhD, ${ }^{a}$ Debin Liu, MD, PhD, ${ }^{\mathrm{b}}$ Feixue Song, MD, ${ }^{\mathrm{a}}$ Bin Li, MD, ${ }^{\text {a }}$ Yongzhu Yang, MD, Shenjun Zhu, MD, PhD, ${ }^{\mathrm{c}}$ and Zhiping Wang, MD, PhD, ${ }^{\mathrm{a}}$ Lanzhou and Beijing, China

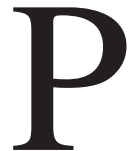

ostpneumonectomy bronchial pleural fistula (PBPF) is one of the most serious complications in cardiac surgery. ${ }^{1}$ Surgical repair of PBPF would be of high risk. The interventional procedures available have limited effects on fistulas with larger orifices. We designed a double-um-

From the Department of Thoracic and Cardiovascular Surgery, ${ }^{a}$ The Second Hospital of Lanzhou University, Lanzhou, China; Department of Surgery, ${ }^{\text {b }}$ Cardiovascular Institute and Fuwai Hospital; The Medical College of Tsinghua University, ${ }^{\mathrm{c}}$ Beijing, China.

Received for publication Jan 9, 2007; revisions received March 14, 2007; accepted for publication March 16, 2007.

Address for reprints: Jian-hua Zhang, MD, PhD, Department of Thoracic and Cardiovascular Surgery, The Second Hospital of Lanzhou University, Lanzhou 730030, China (E-mail: Zhangjianhua68@yahoo.com.cn). Sheng-shou Hu, MD, PhD, Department of Cardiovascular Surgery, Cardiovascular Institute and Fu-Wai Hospital, Beijing 100037, China (E-mail: huss@163bj.com).

J Thorac Cardiovasc Surg 2007;134:531-3

$0022-5223 / \$ 32.00$

Copyright $\odot 2007$ by The American Association for Thoracic Surgery doi:10.1016/j.jtcvs.2007.04.013 brella occluder and used it in 6 patients from April 2002 through November 2006. The results are as follows.

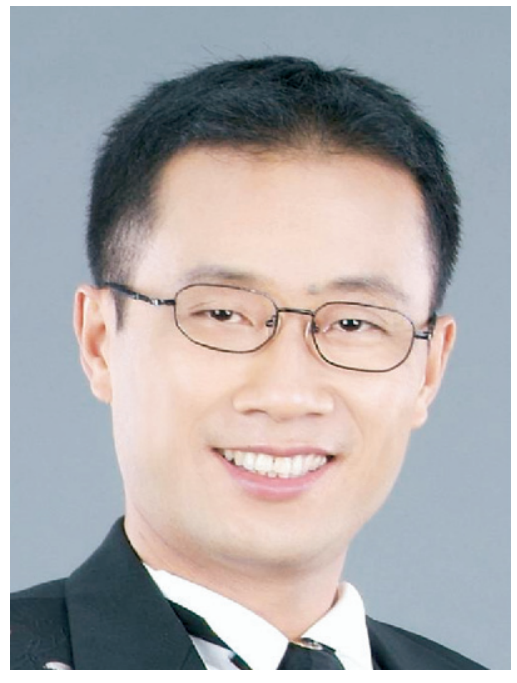

Dr Zhang

\section{Clinical Summary}

A total of 6 patients (4 male and 2 female patients; age range, approximately 34-74 years) were included in this study. Patients had tuberculous thick walled cavity $(n=1)$, tuberculosis-destroyed lungs $(n=2)$, chronic lung abscess $(n=1)$, and central-type lung cancer $(n=2)$. One patient had preoperative radiotherapy, 1 patient had diabetes mellitus and an older age (74 years), and 1 patient had concomitant dyscrasia. A left entire pneumonectomy was performed in 4 patients, and a right entire pnemuonectomy was performed in 2 patients. The bronchial stump was closed by using manual suturing in 4 patients and a suture stapler in 2 patients. Fistulas and empyema occurred on postoperative days 7 to 21 .

The occluder consists of a proximal umbrella, distal umbrella, proximal metal marker, distal metal marker, waist, and proximal nut (Figure 1,A). The internal filler was layers of polyester fabric, 
A

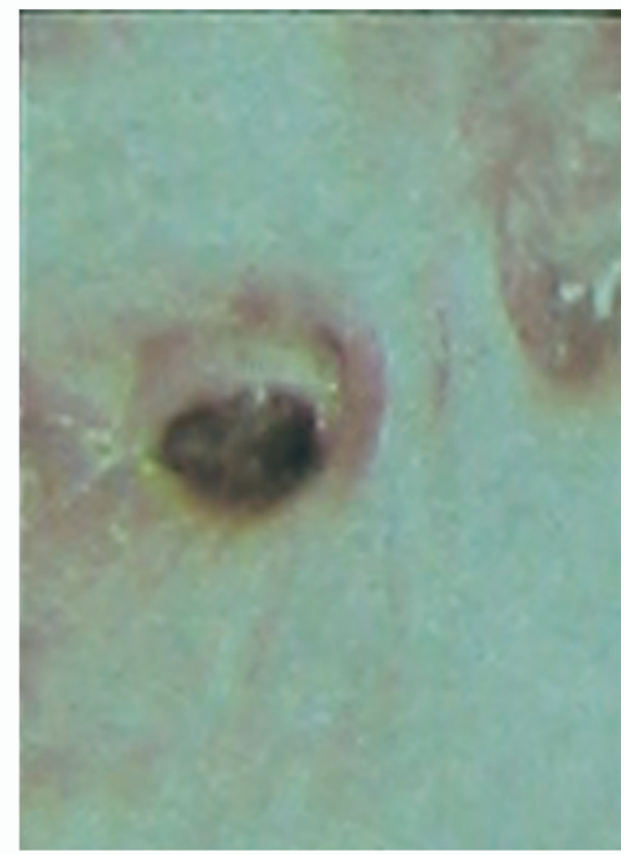

B

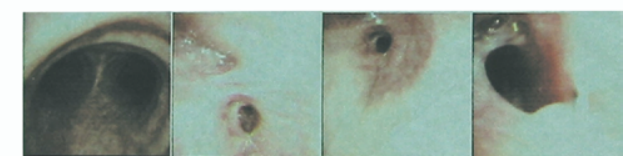

Figure 1. The preoperative preparation of PBPF. A, The bronchoscopic view of PBPF with a 5-mm diameter fistula orifice at the end of the left main bronchus. $B$, The designed draft of the double umbrella-shaped occlusive device and the pushing wire rope. PBPF, Postpneumonectomy bronchial pleural fistula.

and the tectorial membrane was polyurethane. A Shape Memory Alloy Co Ltd manufactured the products.

A fibrobronchoscope was delivered, and then a guide wire was delivered into the orifice of the fistula. The fibrobronchoscope was extracted, with the guide wire left behind. The delivery sheath was delivered, and then the guide wire was extracted. The occluder was pushed in through the delivery sheath with a pusher. Subsequently, the distal umbrella, waist, and proximal umbrella were released. The delivery sheath was withdrawn, with the pusher left in place. The inflation examination was performed to make sure there was no air leakage through the chest tube. After the shape of the double umbrella was determined to be satisfactory by means of fluoroscopy, the pusher was screwed out. Because 1 patient could not tolerate general anesthesia, the procedure was done through an existing chest tube and succeeded.

All patients went back to the ward with chest tubes. Pleural space irrigation was performed with solution containing antibiotic and chymotrypsin. The follow-up included clinical examination, radiography, and fibrobronchoscopy in the first, second, and fourth weeks and every 3 months thereafter.

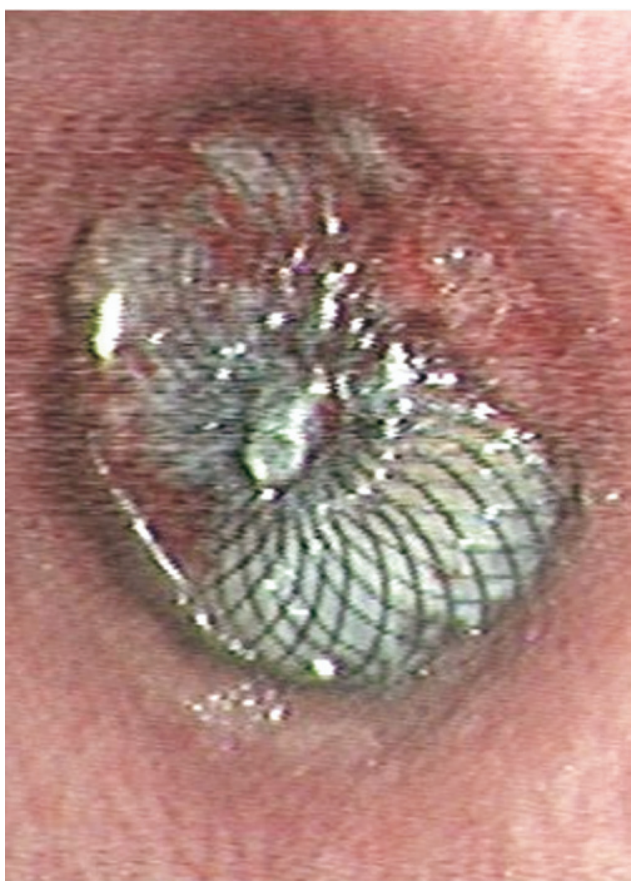

Figure 2. The bronchoscopic view of the PBPF showing that the mucous membrane covered the occlusive device at 1-month follow-up. PBPF, Postpneumonectomy bronchial pleural fistula.

The duration of the operation ranged from 20 to 50 minutes. The operation was performed successfully once in 4 patients and twice in 2 patients to exchange larger occluders. During the procedure, the occluder expanded into a dumbbell shape (Figure 1, B) and the typical double-umbrella shape. The air leakage ceased within 24 hours in 3 patients and 1 week in others. The occluder was covered by a mucous membrane at 30 days (Figure 2). Complete healing of the empyema ranged from 2 to 5 months. No major operation-related complications occurred. No bronchial pleural fistula reoccurred. No patients died because of the recurrence of bronchial pleural fistula. One patient died 2 years after the operation because of the recurrence of lung cancer, and 1 died of dyscrasia 3 months later. The follow-up rate was $100 \%$.

\section{Discussion}

Surgical repair of the bronchial stump of PBPF is restricted because of high risks, serious injury, ${ }^{2}$ and a low success rate.

Some interventional techniques available, including endoscopic burning with a laser or chemistry materials, sclerosing agent injection, fibrin sealant injection, and covered stent placement, have been used because of their convenience, safety, and effectiveness. But they have difficulties in occluding orifice fistulas larger than $3 \mathrm{~mm}$ in diameter. ${ }^{3,4}$

Animal experiments ${ }^{5}$ and our own clinical experiments have shown that interventional occlusion of a PBPF with a specifically designed double-umbrella occluder is a convenient, minimally invasive, economic, and time-saving technique. The occluder has good effect, especially on orifice fistulae of larger than $3 \mathrm{~mm}$ in diameter, and can be localized easily because of its special shape without stenosis and shifting. It neither produces sputum retention 
and abnormal flavor nor induces severe cough. The occluder is small in size, with a definite occluding effect and good histocompatibility. Although the procedure reported here might violate the principle of keeping prosthetic material away from an infected field, this compromise might be appropriate in selected patients. This kind of procedure could be especially useful in the treatment of main bronchus pleural fistulas because it is not easy to shift contrast with a stent. Placement of the occluder through an existing chest tube tract might be developed as a better route because there is no need for general anesthesia and an endotracheal procedure.

\section{Conclusion}

Interventional closure of the PBPF with a specially designed doubleumbrella occluder is a safe, effective, and feasible approach. Because the number of cases is very limited, further studies are needed.

\section{References}

1. Vester SR, Faber LP, Kirtle CF. Bronchopleural fistula after stapled closure of bronchus. Ann Thorac Surg. 1991;52:1253-7.

2. Javadpour H, Sidhu P, Luke DA. Bronchopleural fistula after pneumonectomy. Ir J Med Sci. 2003;172:13-5.

3. Kiriyama M, Fujii Y, Yamakawa Y, et al. Endobronchial neodymium-yttrium-aluminum garnet laser for noninvasive closure of small proximal bronchopleural fistula after lung resection. Ann Thorac Surg. 2002;73:945-8.

4. Hirata T, Ogawa E, Takenaka K, et al. Endobronchial closure of postoperative bronchopleural fistula using vascular occluding coils and n-butyl-2-cyanoacrylate. Ann Thorac Surg. 2002;74:2174.

5. Li Qiang, Yang Xiaoming, Gong Shanshi, et al. Development of bronchial occlusive device made of NiTi memory alloy and its application for bronchial closure in dogs. Acad J Second Milit Med University. 2004;25:697-700.

\title{
Aneurysmal sternal bone cyst: A novel treatment method
}

\author{
Christopher T. Wartmann, MD, Carol D. Morris, MD, Malika Latmore, BS, and Raja M. Flores, MD, New York, NY
}

$\mathrm{T}$ he sternum is an uncommon location for aneurysmal bone cysts (ABCs). We present the case of a 25-yearold wrestler who was treated nonconventionally with curettage and bone graft substitute, negating the need for sternectomy with reconstruction.

\section{Clinical Summary}

This wrestler and athletic trainer was referred to an orthopedist after noting some persisting mild peristernal pain and swelling for 1 month's duration. The patient noted no other symptoms. A magnetic resonance imaging scan of his chest showed a $5.6-\mathrm{cm}$ multiloculated lesion within his lower sternum (Figure 1, B); a computed tomographic scan revealed a soft tissue mass causing lytic destruction of the posterior sternum (Figure 1, A). No other abnormalities were noted. Open biopsy confirmed the diagnosis of $\mathrm{ABC}$, both the solid and cystic types.

The patient underwent open surgical excision of the lesion. An elliptical incision was made over the sternum to incorporate the old incision, and the periosteum was split. An obvious defect in the anterior sternum was observed. The gross lesion was removed by using a combination of curets and pituitary rongeurs. Posteriorly,

From the Department of Surgery, Memorial Sloan-Kettering Cancer Center, New York, NY.

Received for publication March 23, 2007; accepted for publication April 12, 2007.

Address for reprints: Raja M. Flores, MD, Memorial Sloan-Kettering Cancer Center, 1275 York Ave, Room C-879, New York, NY 10021 (E-mail: floresr@mskcc.org).

J Thorac Cardiovasc Surg 2007;134:533-5

$0022-5223 / \$ 32.00$

Copyright $\odot 2007$ by The American Association for Thoracic Surgery doi:10.1016/j.jtcvs.2007.04.011 only the periosteum was intact (Figure 2,A). The defect was filled with an osteoinductive bone graft substitute. A $35 \times 34 \times 60-\mathrm{mm}$ mass was removed. The histopathology was consistent with an ABC. Follow-up imaging shows no evidence of recurrence at 2 years (Figure 2, $B$ ), and the patient has fully returned to wrestling with no complaints.

\section{Discussion}

$\mathrm{ABCs}$ are benign tumors of bone histologically composed of blood-filled cystic spaces separated by fibrous septa that contain an admixture of fibroblasts, variable numbers of osteoclast-type giant cells, and reactive woven bone. ABCs most frequently develop in the metaphyses of long bones and the posterior elements of vertebral bodies yet have been previously described arising in the pelvis and sternum. ${ }^{1}$ Approximately three quarters of cases are observed in persons younger than 20 years and rarely affect persons after 30 years of age. ${ }^{2}$ Radiographically, ABCs appear as a subperiosteal, poorly defined osteolysis elevating the periosteum and possibly leading to erosion of the cortex. Magnetic resonance imaging is helpful in showing the fluid levels within the cyst, as well as its internal septation. ${ }^{1}$

Although primary $\mathrm{ABCs}$ are rare lesions with an incidence of 0.14 per $100,000,{ }^{3}$ several treatment modalities exist, including surgical resection en bloc with the affected bone, curettage with bone grafting, selective arterial embolization, percutaneous injection of a fibrosing agent, and local radiation. Curettage with bone grafting is a widely accepted and published treatment modality in sites including the femur and pelvis. Although the sternum is an uncommon site, the mainstay of surgical treatment consists of a resection through partial sternectomy with reconstruction. The preservation of the posterior periosteum in this case coupled with the morbidity of partial sternectomy drove the authors to perform a sternum-sparing operation because $\mathrm{ABC}$ recurrence is evaded simply by means of complete mar- 